\title{
Suprime-33: Suprime-Cam weak lensing cluster survey
}

\author{
Takashi Hamana \\ National Astronomical Observatory of Japan, Mitaka, Tokyo 181-8588, Japan
}

\begin{abstract}
We present the ability of weak lensing surveys to locate massive clusters. We use both analytic models of dark matter halos and mock weak lensing surveys generated from a large cosmological $N$-body simulation. We find that, for a weak lensing survey with a galaxy number density of $n_{g}=30 \operatorname{arcmin}^{-2}$ with a mean redshift $z=1$, the mean number of galaxy clusters which are expected to be identified is $N(\mathrm{~S} / \mathrm{N}>4)=7$ per $1 \mathrm{deg}^{2}$. Weak lensing surveys thus provide a reasonably efficient way to search for massive clusters. We also describe our project on the weak lensing cluster survey exploiting Suprime-Cam (the wide-field prime focus camera on the $8 \mathrm{~m}$ Subaru telescope), called Suprime-33 and present current status. We also present results from its pilot survey in the Suprime-Cam $2.1 \mathrm{deg}^{2}$ field.
\end{abstract}

\section{Introduction}

Recently, it has become feasible to locate massive clusters directly as density enhancements using weak gravitational lensing. Miyazaki et al. (2002) indeed discovered many clusters in a weak lensing halo survey over a 2.1 square degree field, proving the ability of weak lensing to identify massive clusters. Unlike conventional optical or X-ray selected cluster catalogs, weak lensing cluster catalogs are free from a bias toward the luminous objects because the cluster finding is not based on the flux (or galaxy number density) enhancement but on the projected mass enhancement. This is a great advantage of gravitational lensing. However, lensing has its own disadvantages as every other cluster survey technique (optical, X-ray and Sunyaev-Zel'dovich survey) does; for example, the projection effect from unrelated structures in the same line-of-sight (Hamana, Takada \& Yoshida 2004). Since clusters of galaxies are one of most important cosmological probes, it is of fundamental importance to construct large unbiased samples. Given advantages and disadvantages in each survey technique, it is desirable to combine, in a complementary manner, several techniques to construct an unbiased catalog. Such catalogs provide a valuable data set to investigate, for example, the physical state of the intra-cluster medium. To do this, it is important to correctly understand the selection function and completeness of each survey technique. For this purpose, we examined ability of a weak lensing cluster survey using both analytic models and mock numerical simulations (Hamana et al. 2004). We summaries the basic results from the research in section 3 .

In section 2, we describe our project on the weak lensing cluster survey exploiting Suprime-Cam (the wide-field prime focus camera on the $8 \mathrm{~m}$ Subaru telescope), called Suprime-33 and present current status. We also present results from its pilot survey in the Suprime-Cam $2.1 \mathrm{deg}^{2}$ field.

\section{Suprime-33}

Suprime-33 is a weak lensing survey which exploits the unique capabilities of SuprimeCam. Its primary purpose is to construct, for the first time, a mass selected cluster 
catalog. Conventionally, cluster catalogs were generated by either an optical or X-ray observation. Galaxy clusters in such catalogs are selected based on the (either optical or X-ray) flux enhancement, therefore they must be biased toward luminous objects. Importantly, a weak lensing survey has the potential to detect massive but optical/X-ray faint clusters which conventional surveys have missed.

In our pilot weak lensing cluster search in a GTO $2 \mathrm{deg}^{2}$ field, we had indeed detected 5 clusters with a significant signal ( $/ \mathrm{N}>5$ ) (Miyazaki et al. 2002), which demonstrated that the weak lensing survey is practical with Suprime-Cam. We are currently expanding the survey area to $33 \mathrm{deg}^{2}$ and expect our sample to contain about 100 clusters. We chose the Suprime-33 survey area where ROSAT/PSPC's deep pointings X-ray data $\left(T_{\text {exp }}>25\right.$ ksec) are available. This allows us to construct the mass selected cluster catalog having deep X-ray data.

The primary science goal of Suprime-33 is to test the structure formation paradigm with a well defined mass-limited cluster catalog. One of the most important and unambiguous tests of this paradigm concerns the abundance of halos and its evolution with redshift. These have been extensively studied using $N$-body simulations, and are regarded as well-established theoretically. Therefore, the comparison between the theoretical prediction and our weak lensing measurement is one of the simplest and the most direct method to test the paradigm. This test has not yet been achieved because it requires large observational resources. Also, using this first mass selected cluster catalog which covers a redshifts up to $\sim 0.8$, we will be able to make important contributions to the understanding of cluster physics.

\section{Ability of weak lensing cluster surveys}

The ability of weak lensing cluster surveys to locate massive clusters was examined in detail by Hamana et al. (2004) using numerical simulations. They showed that the expected number of clusters detected in weak lensing surveys is 7 per $1 \mathrm{deg}^{2}$ for a high detection signal of $\mathrm{S} / \mathrm{N}>4$ and for the survey depth of Suprime-33 $\left(R_{\lim }=26\right)$. This is comparable to a deep X-ray selected cluster counts $\left(1\right.$ (5) per $1 \mathrm{deg}^{2}$ for $f_{[0.5-2 \mathrm{keV}]}>$ $1 \times 10^{-13}\left(>2 \times 10^{-14}\right)$ erg $\left.\mathrm{cm}^{-2} \mathrm{~s}^{-1}\right)$. The exposure time to reach $R_{\mathrm{lim}}=26$ is only $30 \mathrm{~min}$ for Suprime-Cam on the $8 \mathrm{~m}$ Subaru telescope, and Suprime-Cam has $27 \times 34$ FOV. Thus a survey cost is 2 hours per $1 \mathrm{deg}^{2}$. Weak lensing surveys thus provide a reasonably efficient way to search for massive clusters.

\section{Acknowledgements}

T.H. acknowledges supports from Japan Society for Promotion of Science (JSPS) Research Fellowships. The $N$-body simulations used in this work (section 3 ) were carried out by the Virgo Consortium at the computer center at the Max-Planck-Institut, Garching. Numerical computations presented in this paper were partly carried out at ADAC (the Astronomical Data Analysis Center) of the National Astronomical Observatory, Japan, and at the Yukawa Institute Computer Facility.

\section{References}

Miyazaki S., et al., 2002, ApJ, 580, L97

Hamana T., Takada M., Yoshida N., 2004, MNRAS, in press 